\title{
Transcranial doppler ultrasonography should it be the first choice for persistent foramen ovale screening?
}

\author{
Monika Komar ${ }^{1 *}$, Maria Olszowska', Tadeusz Przewłocki ${ }^{2}$, Jakub Podolec ${ }^{2}$, Jakub Stępniewski ${ }^{1}$, Bartosz Sobień ${ }^{1}$, \\ Rafał Badacz², Anna Kabłak-Ziembicka², Lidia Tomkiewicz-Pająk and Piotr Podolec ${ }^{1}$
}

\begin{abstract}
Background: Persistent foramen ovale (PFO) is considered a cause of cryptogenic stroke and a risk factor for neurological events in young patients. The reference standard for identifying a PFO is contrast-enhanced transesophageal echocardiography (TEE).

The goal of this study was to evaluate the feasibility of transcranial color Doppler (TCD) and its diagnostic sensitivity compared with TEE.

Methods: We investigated 420 patients admitted to our department with cryptogenic stroke, transient ischemic attacks or other neurological symptoms. All patients underwent TCD and TEE evaluation. TCD and TEE examinations were performed according to a standardized procedure: air-mixed saline was injected into the right antecubital vein three times, while the Doppler signal was recorded during the Valsalva maneuver. During TCD the passage of contrast into the right-middle cerebral artery was recorded 25 seconds following the Valsalva maneuver.

Results: We detected a right-to-left shunt in 220 patients (52.3\%) and no-shunts in 159 patients (37.9\%) with both TCD and TEE. In 20 (4.8\%) patients TEE did not reveal contrast passage which was then detected by TCD. In 21 (5.0\%) patients only TEE revealed a PFO. The feasibility of both methods was 100\%. TCD had a sensitivity of 95\% and a specificity of $92 \%$ in the diagnosis of PFO.

Conclusions: TCD has a relatively good sensitivity and specificity. TCD and TEE are complementary diagnostic tests for PFO, but TCD should be recommended as the first choice for screening because of its simplicity, non-invasive character, low cost and high feasibility.
\end{abstract}

Keywords: Persistent foramen ovale, Transcranial color doppler ultrasound, Transesophageal echocardiography

\section{Background}

Persistent foramen ovale (PFO) is considered a cause of cryptogenic stroke and a risk factor for neurological events in patients under 40 years of age. PFO has been also associated with several disease processes such as arterial gas embolism due to decompression, or platypnea-orthodeoxia syndrome [1-4]. Although the clinical significance of PFO in relation to cryptogenic stroke is still debated, identification and assessment of this abnormality are nowadays a routine diagnostic procedure.

\footnotetext{
*Correspondence: moni_s@interia.pl

'Department of Cardiac and Vascular Diseases, John Paul II Hospital, Institute of Cardiology, Collegium Medicum, Jagiellonian University, Krakow, Poland Full list of author information is available at the end of the article
}

Agitated saline serum has been used in transthoracic echocardiography (TTE), transesophageal echocardiography (TEE) and transcranial color Doppler ultrasound (TCD) for the detection of intracardiac shunts. The reference standard for identifying a PFO is contrast-enhanced TEE but it is a semi-invasive, inconvenient and stressful for the patients and also quite expensive procedure.

Some studies have analyzed the most suitable strategy for the screening, diagnosis and quantification of PFO [5-8]. The aim of this study was to evaluate the feasibility of transcranial Doppler and its diagnostic sensitivity compared with transesophageal echocardiography. 
Table 1 Demographic characteristics of the patients

\begin{tabular}{ll}
\hline & Patients $(\mathbf{n}=\mathbf{4 2 0})$ \\
\hline Obmoking & $205(48.8 \%)$ \\
Diabetes mellitus & $72(17.4 \%)$ \\
Hypertension & $19(4.5 \%)$ \\
Dyslipidemia & $64(15.2 \%)$ \\
\hline
\end{tabular}

\section{Methods}

\section{Patient population}

We investigated 420 consecutive patients [(260 females, 160 males); mean age $34.8 \pm 16.7(13,65)$ years] admitted to our department with cryptogenic stroke, transient ischaemic attacks (TIA) or other neurological symptoms between 2007 and 2014. Most patients (over 50\%) had a history of TIA. The main demographic characteristics of the patients are summarized in Table 1.

\section{Protocol}

All patients underwent TCD and TEE evaluation. The studies were performed on the same day by two different experienced cardiologists, unaware with respect to the result of the other study. The patients were examined in the fasting state. Written informed consent was obtained from all patients. The patients were in left lateral decubitus position during both studies. The studies were performed at resting state and during the Valsalva maneuver after contrast injection. The patients had been trained in performing the Valsalva maneuver before study. The training phase included up to 5 Valsalva maneuver attempts, with at least two resulting in septal shifting or reduced middle cerebral artery flow velocity which was followed by contrast injection and the Valsalva maneuver solicited. The study was repeated at least three times.

\section{Agitated saline contrast test}

TCD and TEE examinations were performed according to a standardized procedure. In brief, $10 \mathrm{~mL}$ of airmixed saline was injected into the right antecubital vein at three different times, while the Doppler signal was recorded during the Valsalva maneuver. During TCD the passage of contrast into the right-middle cerebral artery was recorded 25 seconds after the Valsalva maneuver. A mixture of $9 \mathrm{~mL}$ physiological saline and $1 \mathrm{~mL}$ air was agitated 10 times in $210-\mathrm{mL}$ syringes connected to a 3 way stopcock to exchange the air-saline mix and achieve good dilution. The bolus of saline solution was prepared and injected by the same nurse using the same method in all studies. The patients started the maneuver about 5 seconds after contrast injection, pressing against the closed glottis for at least 10 seconds. They then performed a deep expiration and inspiration, followed by a deep expiration.

\section{Transcranial doppler ultrasonography}

A baseline TCD examination was performed with a Toshiba Power Vision echo-machine using a $2-\mathrm{MHz}$ probe, according to standard practice guidelines. Middle cerebral artery flow was monitored through the temporal bone window. The middle cerebral artery was identified with color Doppler in its proximal portion and insonated bilaterally (Figure 1). On TCD study, the effectiveness of the Valsalva maneuver was verified by a reduction of the middle cerebral artery flow velocity, in comparison with the basal spectrum. TCD was considered positive if at

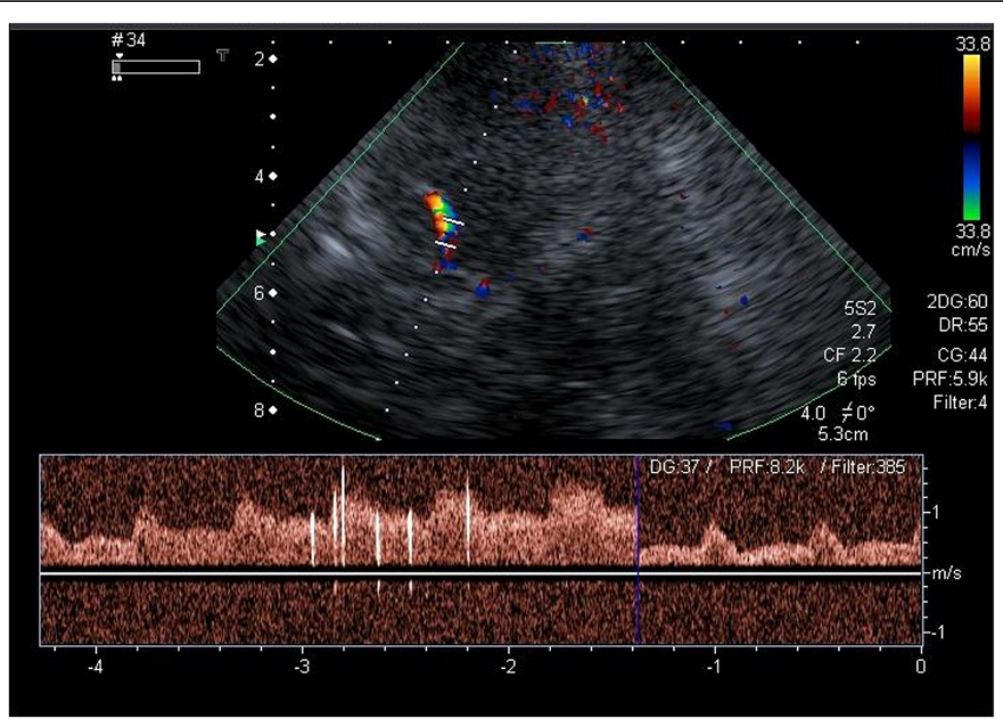

Figure 1 Contrast TCD during the Valsalva maneuver. The middle cerebral artery was identified with color Doppler. The passage of contrast into the right middle cerebral artery (MCA). 
least one microembolic signal was recorded on TCD spectrum within 25 seconds from contrast injection. The shunt was defined as small (1, 10 microembolic signals), medium (>10 microembolic signals), or large ( $>10$ microembolic signals with "curtain").

\section{Transesophageal echocardiography}

The patients received local pharyngeal anesthesia with $10 \%$ topical lidocaine for TEE study. A TEE study was performed using a Toshiba Power Vision machine with a 5.0-MHz multiplane probe, according to a standard protocol including color flow Doppler data. The atrial septum was analyzed from the transverse mid-esophageal four-chamber view to the longitudinal biatrial-bicaval view (Figure 2). Since PFO was not clearly recognized by a bicaval view at $90^{\circ}$ rotation, additional image planes $\left(60^{\circ}, 90^{\circ}\right.$ and/or $110^{\circ}-130^{\circ}$ ) were used to better analyze the atrial septum. On TEE study, the effectiveness of the Valsalva maneuver was verified by a reduction in right ventricular and atrial size and by bulging of the atrial septum into the left atrium. To assess PFO, semi-quantification of the right to left shunt (RLS) was performed. On TEE the evaluation was based on counting the number of micro bubbles (MBs) moving from the right atrium to the left atrium through the PFO after the Valsalva maneuver, within the first three cardiac cycles. The studies were considered positive for PFO when at least one micro bubble was observed in the left atrium. The severity of the shunt was quantified as mild ( $<10$ micro bubbles), moderate (10-20 micro bubbles), or severe $(>20)$. If the quantitative results varied between the first and second study the largest number of MBs decided about the shunt size.

\section{Statistical analysis}

The SPSS 13.0 software package was used for statistical analysis. Quantitative data were expressed as the mean \pm standard deviation, qualitative data as the percentage. The performance indexes used were sensitivity, specificity, predictive positive value (PPV), negative predictive value (NPV), and k concordance index, which express the agreement proportion beyond chance.

Sensitivity was defined as:

$$
\text { sentivity }=\frac{\text { number of True Positives }}{\text { number of True Positives }+ \text { number of False Negatives }}
$$

Specificity was defined as:

$$
\text { specificity }=\frac{\text { number of True Negatives }}{\text { number of True Negatives }+ \text { number of False Positives }}
$$

\section{Results}

Using both TCD and TEE, we detected a right-to-left shunt in 220 patients (52.3\%) and no-shunts in 159 patients (37.9\%). In 20 (4.8\%) patients TEE did not show the passage of contrast which was later detected by TCD. In 21 (5.0\%) patients only TEE revealed a PFO. The feasibility of both methods was $100 \%$. Shunts confirmed by both methods (TCD and TEE) were found in: $78.4 \%$ of patients with cryptogenic stroke, in $48.6 \%$ of patients with TIA, and only in $10.4 \%$ of patients with migraine without aura (Table 2). Considering TEE as the gold standard technique, TCD was able to identify 200 (91.3\%) of the 241 patients with PFO. TCD showed a NPV of $89 \%$, PPV of $98 \%$, sensitivity of $95 \%$, and specificity of $92 \%$. TCD showed a high concordance $(k=0.89)$

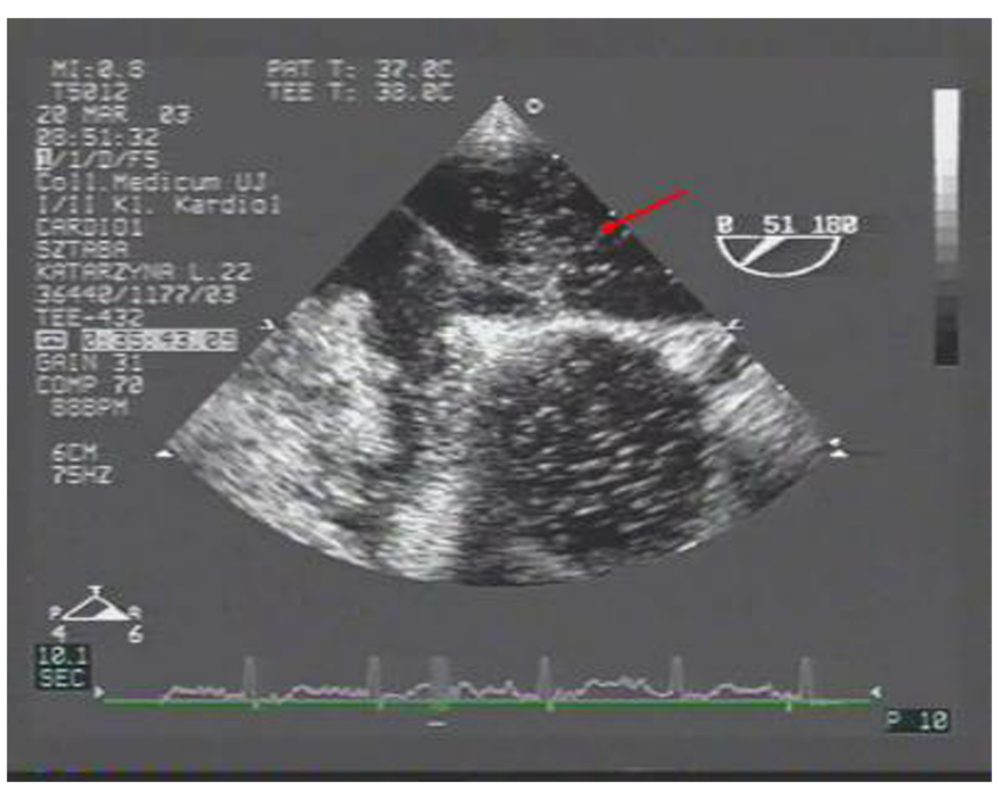

Figure 2 Contrast TEE during the Valsalva maneuver. Contrast agent in the left atrium. 
Table 2 Shunt grading using both transcranial doppler ultrasonography and transesophageal echocardiography

\begin{tabular}{llll}
\hline SHUNT & $\begin{array}{l}\text { TEE (N=220 pts - } \\
\text { TEE and } \\
\text { TCD positive) }\end{array}$ & $\begin{array}{l}\text { TCD (N=220 pts - } \\
\text { TEE and } \\
\text { TCD positive) }\end{array}$ & $\begin{array}{l}\text { \% of the same } \\
\text { grading using } \\
\text { both methods }\end{array}$ \\
\hline Small & $56(25.5 \%)$ & $70(31.8 \%)$ & 62.1 \\
Medium & $123(55.9 \%)$ & $119(54.1 \%)$ & 68.3 \\
Large & $41(18.6 \%)$ & $31(14.1 \%)$ & 83.2 \\
\hline
\end{tabular}

TCD Transcranial Doppler Ultrasonography, TEE Transesophageal Echocardiography.

with TEE in PFO recognition (Table 3). Shunt grading in both TCD and TEE methods was classified with the same score in $70.5 \%$ of cases, and it was the highest in the large shunt (Table 4).

\section{Discussion}

Patent foramen ovale is a hemodynamically trivial interatrial communication that is present in about $25 \%$ of the adult population. However, the clinical significance of PFO in relation to cerebrovascular events is still debated, identification and assessment of this abnormality are nowadays a routine diagnostic procedure [6,9-12]. The search for PFO is becoming more and more common in young subjects suffering from migraine, TIA or cryptogenic stroke [12-17]. At the moment there is no clearly recognized standard protocol for performing both TEE and TCD studies to identify PFO. For the first time, based on the available literature, we developed a standardized exercise protocol in both TEE and TCD studies.

Contrast transesophageal echocardiography is the gold standard for the diagnosis of PFO, regardless of the fact that this procedure is semi-invasive [18-24]. Therefore, transcranial Doppler ultrasonography seems to be an attractive alternative to TEE in the recognition of PFO, because it is a cheap and noninvasive examination [25-29].

The aim of this study was to evaluate the feasibility of transcranial Doppler and its diagnostic sensitivity compared with transesophageal echocardiography. Although the diagnostic power of TCD has been reported, only a few studies

Table 3 Identification of persistent for amen ovale using both transcranial color doppler and transesophageal echocardiography

\begin{tabular}{ccc}
\hline \multirow{2}{*}{$\begin{array}{c}\text { Clinical characteristics } \\
\text { of patients }\end{array}$} & \multicolumn{2}{c}{$\begin{array}{c}\text { Identification of PFO } \\
\text { (both TCD and TEE) }\end{array}$} \\
\cline { 2 - 3 } & Patients (n = 420) & Patients $(\mathbf{n}=\mathbf{2 2 0})$ \\
\hline TIA & $218(51.9 \%)$ & $106 / 218(48.6 \%)$ \\
Migraine & $67(15.6 \%)$ & $7 / 67(10.4 \%)$ \\
Migraine with aura & $50(11.9 \%)$ & $38 / 50(76.0 \%)$ \\
Cryptogenic stroke & $88(20.9 \%)$ & $69 / 88(78.4 \%)$ \\
\hline
\end{tabular}

PFO Patent Foramen Ovale, TCD Transcranial Doppler Ultrasonography, TEE Transesophageal Echocardiography, TIA Transient Ischaemic Attack.
Table 4 Sensitivity, specificity, negative predictive value, and positive predictive value of transcranial doppler ultrasonography in comparison with transesophageal echocardiography in pfo detection

\begin{tabular}{llllll}
\hline Technique & $\begin{array}{l}\text { PFO } \\
\text { (\%) }\end{array}$ & $\begin{array}{l}\text { PPV } \\
\text { (\%) }\end{array}$ & $\begin{array}{l}\text { NPV } \\
\text { (\%) }\end{array}$ & $\begin{array}{l}\text { Sensitivity } \\
\text { (\%) }\end{array}$ & $\begin{array}{l}\text { Specificity } \\
\text { (\%) }\end{array}$ \\
\hline $\begin{array}{l}\text { Contrast- } \\
\text { enhanced TCD }\end{array}$ & 90 & 98 & 89 & 95 & 92
\end{tabular}

NPV: Negative Predictive Value, PPV: Negative Predictive Value TCD: Transcranial Doppler Ultrasonography, TEE: Transesophageal Echocardiography, PFO: Patent Foramen Ovale.

comparing both techniques (TCD and TEE) have been published [5,30-33]. In our study TCD had a good sensitivity and specificity. In our opinion, in contrast to other investigators, TCD and TEE are complementary for PFO diagnosis, but TCD should be recommended as the first choice for screening because of its simplicity, non-invasive character, low cost, and high feasibility. Contrary to other researchers, our results and clinical experience show that both methods are necessary and complementary to recognize a significant foramen ovale.

The sensitivity and specificity of TCD for shunt detection vary in different studies according to both protocol and diagnostic criteria [25-33]. In our study TCD had 89\% NPV, 98\% PPV, 95\% sensitivity, and 92\% specificity. In the Caputi study a general concordance of up to $90 \%$ between TEE and TCD was found. TCD sensitivity and specificity were $96.8 \%$ and $78.4 \%$, respectively [25].

Several studies have suggested that the low specificity of TCD may be due to the fact that TCD is able to detect both cardiac and pulmonary shunts [5]. The specificity for a PFO shunt may be increased by a defined time of micro bubble appearance. The passage time from the antecubital vein injection site to the middle cerebral artery (MCA) through an intracardiac shunt is about 11 seconds, and it is about 14 seconds in the case of pulmonary passage. The overlap interval between intrapulmonary and cardiac passage level must be taken into account [33].

In our study only in $4.8 \%$ of patients TEE did not show the passage of contrast which was later detected by TCD. The meaning of a shunt detected only during TCD is not clearly understood, but a very small intracardiac shunt, not detected by TEE, cannot be excluded. Furthermore the Valsalva maneuver may be less effective during TEE than during TCD, because of esophageal intubation. It is crucial to perform the Valsalva maneuver properly. Contrary to other investigators, in our material, the patients had been trained in performing the Valsalva maneuver before study.

Recent studies as well as our results (95\% sensitivity, and $92 \%$ specificity of TCD), show a good concordance between TCD and TEE with sensitivity ranging from $70 \%$ to $100 \%$ and specificity $>95 \%[27,31,32]$. 
Both techniques have class IIA recommendations for shunt detection [33].

Taking into account that TCD and TEE have similar sensitivity and specificity in shunt detection TCD should be recommended as the first choice for screening because of its low cost, and high feasibility. TCD is also a noninvasive exam - easy to perform and repeat, if necessary. TEE should be limited to the patients scheduled for transcatheter PFO closure, patients with high-risk PFO with recurrent stroke, and patients with ASA or large shunt detected on TCD.

\section{Conclusion}

TCD has a relatively good sensitivity and specificity. TCD and TEE are complementary for PFO diagnosis, but TCD should be recommended as the first choice for screening because of its simplicity, non-invasive character, low cost, and high feasibility.

\section{Abbreviations}

MBs: Micro bubbles; MCA: Middle cerebral artery; NPV: Negative predictive value; PFO: Persistent foramen ovale; PPV: Positive predictive value; RLS: Right to left shunt; TCD: Transcranial color doppler; TEE: Transesophageal echocardiography; TIA: Transient ischaemic attack; TTE: Transthoracic echocardiography.

\section{Competing interests}

The authors declare that they have no competing interests.

\section{Authors' contributions}

MK: contributed to study conception and design, acquisition of data, analysis and interpretation of data, drafting the manuscript, revising it critically for important intellectual content. MO: contributed to study conception and design, helped to draft the manuscript, revising it critically. TP: contributed to study conception and design, acquisition of data, analysis and interpretation of data, drafting the manuscript, revising it critically for important intellectual content, gave final approval of the version to be published. JP: contributed to study conception and design, analysis and interpretation of data. JS: analysis and interpretation of data, drafting the manuscript. BS: contributed to study conception and design, acquisition of data, analysis and interpretation of data, drafting the manuscript. RB: contributed in revising critically for important intellectual content. AKZ: analysis and interpretation of data, revising it critically for important intellectual content. LTP: contributed in acquisition of data, analysis and interpretation of data, drafting the manuscript, revising it critically for important intellectual content. PP: contributed to study conception and design, revising it critically for important intellectual content, gave final approval of the version to be published. All author's read approved the final manuscript.

\section{Author details}

${ }^{1}$ Department of Cardiac and Vascular Diseases, John Paul II Hospital, Institute of Cardiology, Collegium Medicum, Jagiellonian University, Krakow, Poland. ${ }^{2}$ Department of Interventional Cardiology, John Paul II Hospital, Institute of Cardiology, Jagiellonian University Medical College, Krakow, Poland.

\section{Received: 15 April 2014 Accepted: 2 May 2014}

\section{Published: 22 May 2014}

\section{References}

1. Gupta V, Yesilbursa D, Huang WY, Aggarwal K, Gupta V, Gomez C, Patel V, Miller AP, Nanda NC: Patent foramen ovale in a large population of ischemic stroke patients: diagnosis, age distribution, gender, and race. Echocardiography 2008, 25:217-227.

2. Carerj S, Narbone MC, Zito C, Serra S, Coglitore S, Pugliatti P, Luzza F, Arrigo F, Oreto $G$ : Prevalence of atrial septal aneurysm in migraine patients: an echocardiographic study. Headache 2003, 43:725-728.
3. De Castro S, Cartoni D, Fiorelli M, Rasura M, Anzini A, Zanette EM, Beccia M, Colonnese C, Fedele F, Fieschi C, Pandian NG: Morphological and functional characteristics of patent foramen ovale and their embolic implications. Stroke 2000, 31:2407-2413.

4. Homma S, Sacco R, Di Tullio M, Sciacca R, Mohr J: Effect of medical treatment in stroke patients with patent foramen ovale: patent foramen ovale in Cryptogenic Stroke Study. Circulation 2002, 105:2625-2631.

5. Khalid MM, Scott RC, Jared WS, Jorge R, David G-M, Rubine G, Tobis JM: Accuracy of transcranial Doppler for the diagnosis of intracardiac rightto-left shunt: a bivariate meta-analysis of prospective stusies. JACC Cardiovas Imaging 2014, 7(3):236-250.

6. Hara H, Virmani R, Ladich E, Mackey-Bojack S, Titus J, Reisman M, Gray W, Nakamura M, Mooney M, Poulose A, Schwartz RS: Patent foramen ovale: current pathology, pathophysiology, and clinical status. J Am Coll Cardiol 2005, 46:1768-1776.

7. Kerut EK, Norfleet WT, Plotnick GD, Giles TD: Patent foramen ovale: a review of associated conditions and the impact of physiological size. J Am Coll Cardiol 2001, 38:613-623.

8. Di Tullio MR, Sacco RL, Sciacca RR, Jin Z, Homma S: Patent foramen ovale and the risk of ischemic stroke in a multiethnic population. J Am Coll Cardiol 2007, 49:797-802.

9. Krumsdorf U, Ostermayer S, Billinger K, Trepels T, Zadan E, Horvath K, Sievert $\mathrm{H}$ : Incidence and clinical course of thrombus formation on atrial septal defect and patient foramen ovale closure devices in 1,000 consecutive patients. J Am Coll Cardiol 2004, 43:302-309.

10. Woods T, Patel A: A critical review of patent foramen ovale detection using saline contraste echocardiography: when bubbles lie. J Am SoC Echocardiogr 2006, 19:215-222.

11. Zito C, Dattilo G, Oreto G, Di Bella G, Lamari A, ludicello R, Trio O, Caracciolo G, Coglitore S, Arrigo F, Carerj S: Patent foramen ovale: comparison among diagnostic strategies in cryptogenic stroke and migraine. Echocardiography 2009, 26:495-502.

12. Hausmann D, Mügge A, Becht I, Daniel WG: Diagnosis of patent foramen ovale by transesophageal echocardiography and association with cerebral and peripheral embolic events. Am J Cardiol 1992, 70:668-672.

13. Kerut EK, Lee S, Fox E: Diagnosis of an anatomically and physiologically significant patent foramen ovale. Echocardiography 2006, 23:810-815.

14. Mesa D, Franco M, Suárez de Lezo J, Muñoz J, Rus C, Delgado M: Prevalencia de foramen oval permeable en pacientes jóvenes con accidente isquémico cerebral de causa desconocida. Rev Esp Cardiol 2003, 56:662-668.

15. Calvert PA, Rana BS, Kydd AC, Shapiro LM: Patent foramen ovale: anatomy, outcomes, and closure. Nat Rev Cardiol 2011, 8:148-160.

16. Hamilton-Craig C, Sestito A, Natale L, Meduri A, Santangeli P, Infusino F, Pilato F, Di Lazzaro V, Crea F, Lanza GA: Contrast transoesophageal echocardiography remains superior to contrast-enhanced cardiac magnetic resonance imaging for the diagnosis of patent foramen ovale. Eur J Echocardiogr 2011, 12:222-227.

17. Schneider B, Zienkiewicz T, Jansen V, Hofmann T, Noltenius H, Meinertz T: Diagnosis of patent foramen ovale by transesophageal echocardiography and correlation with autopsy findings. Am J Cardiol 1996, 77:1202-1209.

18. Pepi M, Evangelista A, Nihoyannopoulos P, Flachskampf FA, Athanassopoulos G, Colonna P: Recommendations for echocardiography use in the diagnosis and management of cardiac sources of embolism. Eur J Echocardiogr 2010, 11:461-476.

19. Kühl HP, Hoffmann R, Merx MW, Franke A, Klötzsch C, Lepper W, Reineke T, Noth J, Hanrath P: Transthoracic echocardiography using second harmonic imaging: diagnostic alternative to transesophageal echocardiography for the detection of atrial to left shunt in patients with cerebral embolic events. J Am Coll Cardiol 1999, 34:1823-1830.

20. Monte I, Grasso S, Licciardi S, Badano LP: Head-to-head of real-time threedimensional transthoracic echocardiography with transthoracic and transesophageal two-dimensional contrast echocardiography for the detection of patent foramen ovale. Eur J Echocardiogr 2010, 11:245-249.

21. Pearson AC, Labovitz AJ, Tatineni S, Gomez CR: Superiority of transesophageal echocardiography in detecting cardiac source of embolism in patients with cerebral ischemia of uncertain etiology. $J \mathrm{Am}$ Coll Cardiol 1991, 17:66-72.

22. Albers GW, Comess KA, DeRook FA, Bracci P, Atwood JE, Bolger A, Hotson J: Transesophageal echocardiography findings in stroke subtypes. Stroke 1994, 25:23-28. 
23. Teague SM, Sharma MK: Detection of paradoxical cerebral echo contrast embolization by transcranial Doppler ultrasound. Stroke 1991, 22:740-745.

24. Devuyst G, Piechowski-Józwiak B, Karapanayiotides T, Fitting JW, Kémeny V, Hirt L, Urbano LA, Arnold P, van Melle G, Despland PA, Bogousslavsky J: Controlled contrast transcranial Doppler and arterial blood gas analysis to quantify shunt through patent foramen ovale. Stroke 2004, 35:859-863.

25. Caputi L, Carriero MR, Falcone C, Parati E, Piotti P, Materazzo C, Anzola GP: Transcranial Doppler and transesophageal echocardiography: comparison of both techniques and prospective clinical relevance of transcranial Doppler in patent foramen ovale detection. J Stroke Cerebrovasc Dis 2009, 18:343-348.

26. Klötzsch C, Janssen G, Berlit P: Transesophageal echocardiography and contrast TCD in the detection of a patent foramen ovale: experiences with 111 patients. Neurology 1994, 44:1603-1606.

27. Devuyst G, Despland PA, Bogousslavsky J, Jeanrenaud X: Complementarity of contrast transcranial Doppler and contrast transesophageal echocardiography for the detection of patent foramen ovale in stroke patients. Eur Neurol 1997, 38:21-25.

28. Souteyrand G, Motreff P, Lusson JR, Rodriguez R, Geoffroy E, Dauphin C, Boire JY, Lamaison D, Cassagnes J: Comparison of transthoracic echocardiography using second harmonic imaging, transcranial Doppler and transesophageal echocardiography for the detection of patent foramen ovale in stroke patients. Eur J Echocardiogr 2006, 7:147-154.

29. Droste DW, Lakemeier S, Wichter T, Stypmann J, Dittrich R, Ritter M: Optimizing the technique of contrast transcranial Doppler ultrasound in the detection of right-to-left shunts. Stroke 2002, 33:2211-2216.

30. Van H, Poommipanit P, Shalaby M, Gevorgyan R, Tseng $\mathrm{CH}$, Tobis J: Sensitivity of transcranial Doppler versus intracardiac echocardiography in the detection of right-to-left shunt. JACC Cardiovasc Imaging 2010, 3:343-348.

31. Fox ER, Picard MH, Chow CM, Levine RA, Schwamm L, Kerr AJ: Interatrial septal mobility predicts larger shunts across patent foramen ovale: An analysis with transmitral Doppler scanning. Am Heart J 2003, 145:730-736

32. Droste DW, Silling K, Stypmann J, Grude M, Kemény V, Wichter T, Kühne K, Ringelstein EB: Contrast transcranial Doppler ultrasound in the detection of right to left shunts: Time window and threshold in microbubble numbers. Stroke 2000, 31:1640-1645.

33. Sloan MA, Alexandrov AV, Tegeler CH, Spencer MP, Caplan LR, Feldmann E, Wechsler LR, Newell DW, Gomez CR, Babikian VL, Lefkowitz D, Goldman RS, Armon C, Hsu CY: Goodin DS Assessment: Transcranial Doppler ultrasonography: report of the therapeutics and technology assessment subcommittee of the American Academy of Neurology. Neurology 2008, 62:1468-1481

doi:10.1186/1476-7120-12-16

Cite this article as: Komar et al:: Transcranial doppler ultrasonography should it be the first choice for persistent foramen ovale screening? Cardiovascular Ultrasound 2014 12:16.

\section{Submit your next manuscript to BioMed Central and take full advantage of:}

- Convenient online submission

- Thorough peer review

- No space constraints or color figure charges

- Immediate publication on acceptance

- Inclusion in PubMed, CAS, Scopus and Google Scholar

- Research which is freely available for redistribution 